\title{
Baumkronenwelten Teil 2: \\ Epiphytische Aronstabgewächse: Kleine und ganz große
}

\author{
Michael SChWerdtfeger
}

\begin{abstract}
In the Araceae house of the Old Botanical Garden of the University of Göttingen several tropical plants and epiphytic Araceae are cultivated. Their diversity and especially some species of the genera Philodendron and Anthurium are mentioned.
\end{abstract}

\section{Zusammenfassung}

Im Araceen-Haus des Alten Botanischen Gartens der Universität Göttingen werden neben einigen anderen tropischen Pflanzenfamilien viele epiphytische Araceen gezeigt. Ihre Vielfalt und vor allem auch verschiedene Arten der Gattungen Philodendron und Anthurium werden vorgestellt.

\section{Philodendron, Anthurium und die Nostalgie der Plant Hunters}

Eines des charmantesten, stimmungsvollsten historischen Gewächshäuser des Alten Botanischen Gartens der Universität Göttingen ist das Araceenhaus. Dabei wurde dieses Haus um 1830 ,illegal“ gebaut, denn es existiert eine Beschwerde des damaligen Direktors Prof. Schra-

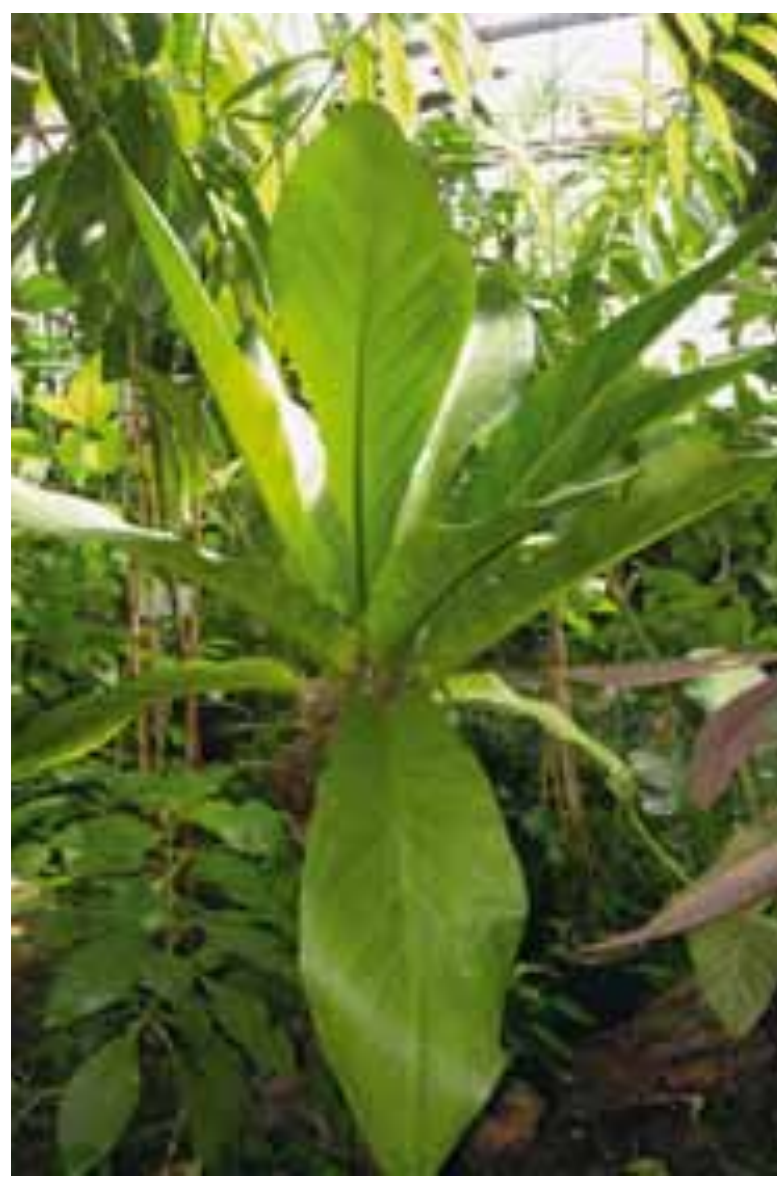

DER, dass das Haus ohne seine Genehmigung und Unterschrift errichtet sei und man derartigem „Unterschleif“ fortan begegnen müsse.

Seit Jahrzehnten sind in diesem feuchtwarmen Haus Pflanzen der tropischen Tieflandregenwälder untergebracht, also Nepenthes, Hoya, Dischidia, Medinilla, Miconia und andere Melastomataceae. Zudem sind dort buntblättrige Maranten und kostbare epiphytische Farne anzutreffen. Die hellen, oberen Bereiche unter dem Glas werden zunehmend für Orchideen genutzt, die hier in Blockkultur hervorragend gedeihen.

Den Schwerpunkt bilden allerdings tropische Aronstabgewächse und unter diesen wiederum die beiden wichtigsten epiphytischen Gattungen der amerikanischen Tropen: Philodendron und Anthurium.

Die Gattung Philodendron ist jedem bekannt, auch wenn der "Zimmerphilodendron" bzw. das „Fensterblatt“ der nahe verwandten Gattung Monstera angehört. Mit ihren ornamentalen, großen und oft schön geformten Blättern gehören die Philodendron-Arten so sehr zu unserer Vorstellung von den lianen- und epiphytenbesetzten Bäumen des tropischen Waldes, dass auf vielen der stimmungsvollen Stahlstiche des 19. Jahrhunderts auch Philodendren zu erkennen sind. Seit Mitte des 19. Jahrhunderts, der

Abb. 1: Anthurium acaule.

Abb. 2 (Seite 13 oben): Anthurium andicola, fruchtend.

Abb. 3 (Seite 13 unten): Anthurium armeniense, blühend. 
Zeit der „Plant Hunters“, gelangten zahlreiche Arten in Kultur und schmückten die Gewächshäuser botanischer Gärten und die Ward'schen Kästen, Pflanzenvitrinen und Gewächshäuser der wohlhabenden Pflanzensammler.

\section{Vielfalt der „Baumfreunde“}

Die meisten der ca. 350 Arten des „Baumfreundes" (so die wörtliche Übersetzung der griechischen Worte philos und dendron) wachsen als Lianen. Die Blätter sind durch lange Internodien getrennt. Mit meterlangen Trieben erklettern die Pflanzen die Baumstämme und heften sich mit Haftwurzeln fest, die an den Knoten in ganzen Kränzen erscheinen. Bei vielen Arten finden wir einen Blatt-Dimorphismus in der Weise, dass die Blätter zarter Jungpflanzen sich von denen erwachsener, in vollem Licht gewachsener blühender Triebe unterscheiden. Eine kleine Gruppe südbrasilianischer Arten wächst mehr oder weniger stehend, so das bekannte Philodendron bipinnatifidum ( $=P$ h. selloum). Interessanter sind aber für unser Thema Baumkronenwelten die halbepiphytischen und epiphytischen Arten. Bei alten, ausgepflanzten Philodendren in unseren Gewächshäusern können wir beobachten, was auch in der Natur regelmäßig passiert: Der "Philo“ entwickelt Massen von Haftwurzeln, die sich dem Trägerbaum anschmiegen, und Luftwurzeln, die freischwebend Feuchtigkeit aufnehmen oder wieder Kontakt zum Erdboden finden und die Pflanze ernähren. Es fällt dann kaum ins Gewicht, dass die ältesten Teile oft längst abgestorben sind und die Pflanze also ihre ursprüngliche Verbindung zum Substrat längst aufgegeben hat. Aus der Kletterpflanze (Liane) ist ein Halb-Aufsitzer (Hemi-Epiphyt) geworden.

Die Aronstabgewächse gehören zu den einkeimblättrigen Pflanzen, denen, von wenigen Ausnahmen abgesehen, die Fähigkeit zum sekundären Dickenwachstum fehlt. Aus dem gleichen Grund fehlt den Einkeimblättrigen aber auch die Hauptwurzel; vielmehr liegt sekundäre Homorrhizie vor, also eine Bewurzelung aus gleichrangigen sprossbürtigen Wurzeln. Ein ausgewachsener Philodendron ist oft ein dreidimensionales, freischwebend im Kronendach kletterndes Raumgebilde aus Blättern,
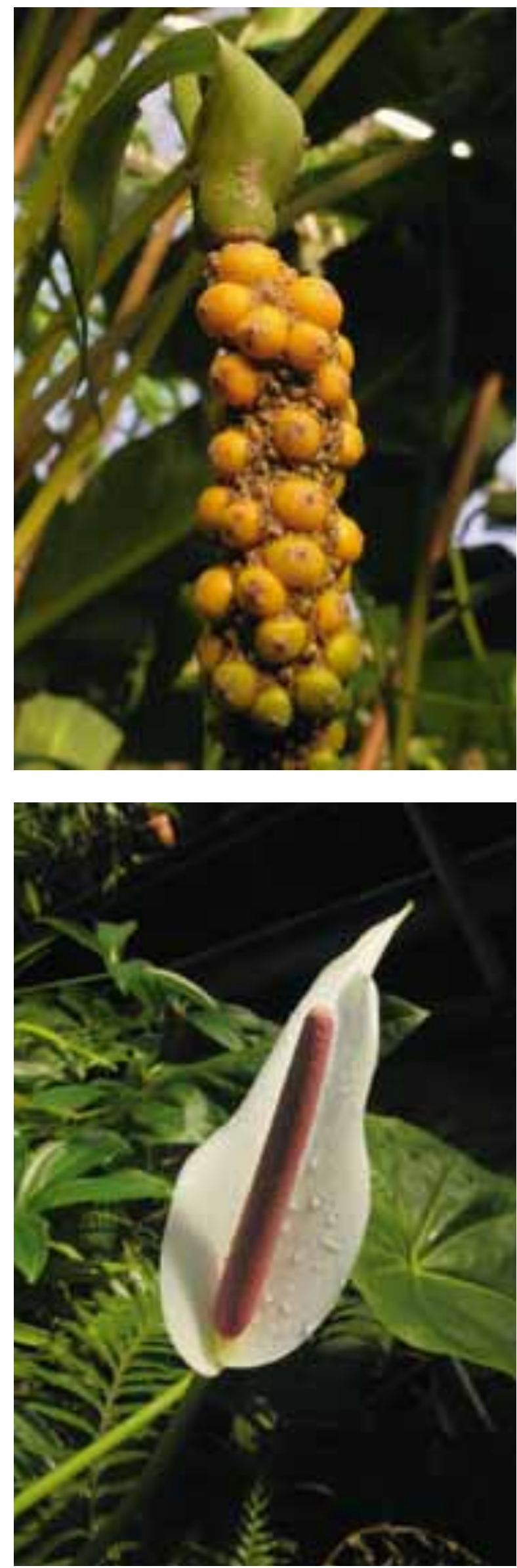


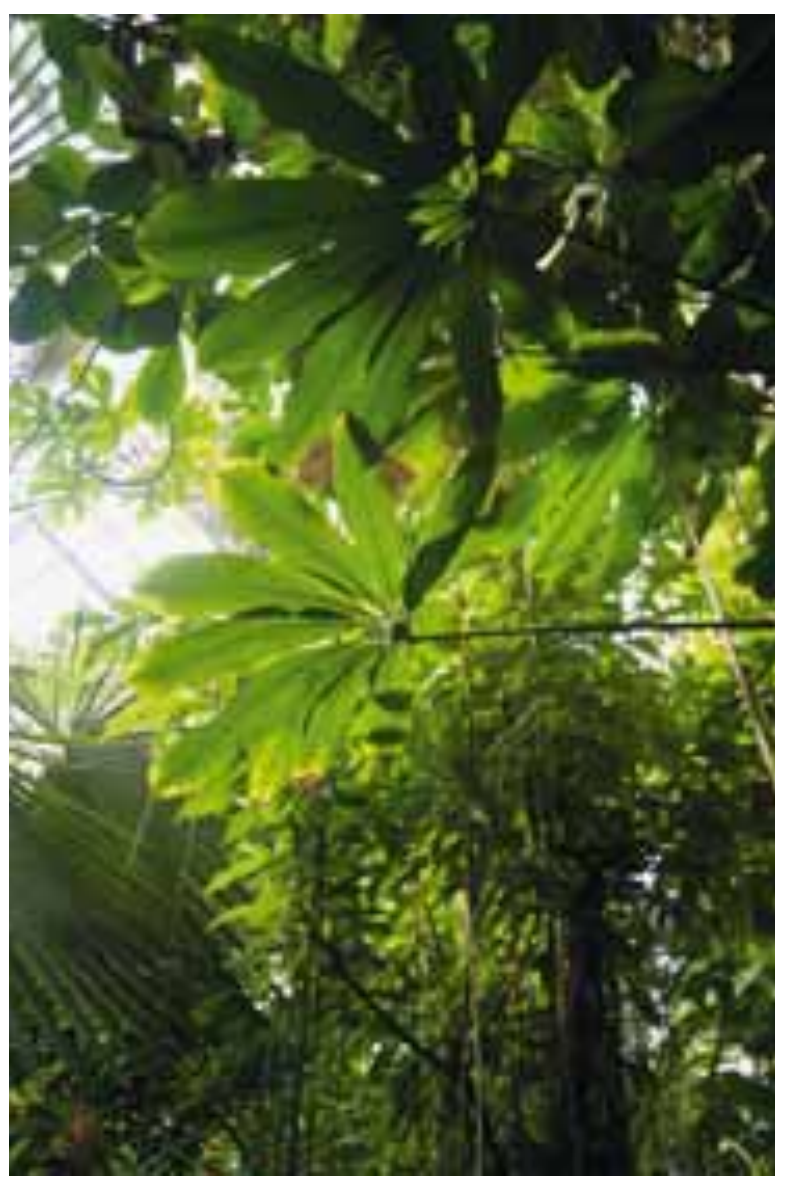

Sprossachsen und Wurzeln, aber ohne deutlichen Stamm oder nennenswerte Basis.

\section{Hoch hinaus: Von der Liane zum Epiphyten}

Von den Hemi-Epiphyten, die ihre Laufbahn am Erdboden beginnen und später den Bodenkontakt lösen, ist es nicht weit zu den echten Epiphyten. Dazu gehört zum Beispiel das wenig bekannte Philodendron campii: Hier sind die Internodien nur kurz, und die Blätter sind daher in einer formschönen, trichterförmigen Rosette angeordnet, gerade so wie bei dem Vogelnestfarn (Asplenium nidus). Genau wie bei diesem sammeln sich im Zentrum der Trichterrosette Falllaub und andere organische Abfallstoffe, die zu einem blumenerdeartigen, humosen Substrat verrotten, von Wurzeln durchzogen werden und dem Epiphyten auf seinem luftigen Sitz Nährstoffzufuhr bescheren.

Besonders interessant sind Arten wie Philodendron martianum (syn. Ph. cannifolium) und $\mathrm{Ph}$. wendlandii, die ebenfalls nicht meterlang klettern, sondern mit kurzer Sprossachse als Epiphyten auf den Ästen nisten. Bei diesen Arten sind die Blattspreiten von lanzettlicher Form, dafür sind die Blattstiele zylindrisch aufgedunsen und erinnern an die Triebe von Cattleya mit ihren Pseudobulben.

\section{Die größte Gattung der Familie: Anthurium in tausend Arten!}

Die beschriebene Vielfalt innerhalb der Gattung Philodendron wird jedoch noch um vieles von der Gattung Anthurium übertroffen. Diese ist mit ca. 1000 Arten die bei Weitem größte der Familie der Aronstabgewächse. Die Verbreitung der Gattung erstreckt sich von Mexiko und der Karibik über das Kerngebiet der neotropischen Regenwälder bis nach Südbrasilien und Bolivien. Die meisten Arten finden wir im Feuchten und Warmen; nur wenige Arten (z. B. Anthurium nelsonii) strahlen in die Trockengebiete aus. Auch in die kühlen Bergregenwälder steigen nur wenige Arten auf, sodass die in den Anden wachsenden Anthurium pulchrum und A. gualeanum die Ausnahme bilden.

In vielen neotropischen Tieflandgebieten wachsen 5-10 Anthurium-Arten sympatrisch nebeneinander, die aber in Wuchs, Blüten- und Verbreitungsökologie jeweils verschiedene ökologische Nischen besetzen. Wer die beiden rot blühenden Arten von Flamingoblumen, also Anthurium scherzerianum und $A$. andraeanum vor Augen hat und damit meint, eine repräsentative Vorstellung von der Gattung zu haben, der irrt: Das für alle Aronstabgewächse so typische Hochblatt (Fahne, Spatha) ist nur bei den genannten Arten so leuchtend rot gefärbt, bei einer Handvoll weiterer weiß (A. antioquiense, $A$. armeniense, $A$. huixtlense), rosa (A. lindenianum), violett (A. amnicola) oder gelb-braun gestreift (A. flavolineatum). Die große Mehrheit der Arten hat jedoch unscheinbare, wenig auf-

Abb. 4 (oben): Blatt von Anthurium digitatum.

Abb. 5 (Seite 15 oben): Anthurium draconopterum.

Abb. 6 (Seite 15 unten links): Anthurium lindenianum mit rosafarbener Spatha.

Abb. 7 (Seite 15 unten rechts): Anthurium loretense. 
fallende Hochblätter. Die Bestäubung der Anthurien wie überhaupt vieler Aronstabgewächse wird nämlich von kleinen Fliegen oder Mücken (Drosophilidae, Cecidomyiidae, Psychodidae etc.) vollzogen, die sich geruchlich orientieren und für die der Sehsinn nur eine geringe Rolle spielt. Entsprechend vielfältig sind die Gerüche der Blütenstände: Die von Obstfliegen bestäubten Arten riechen deutlich nach gärendem Obst, während sich viele Arten als für unsere Nasen geruchlos präsentieren. Aber auch sie müssen geheime Duftbotschaften aussenden, denn in der Natur sind die Blütenstände nachts manchmal von ganzen Versammlungen von Cecidomyiiden bedeckt. Einige Arten produzieren sogar Parfümduftstoffe und werden nach Art der Orchideengattungen Stanhopea, Gongora, Acineta etc. von Prachtbienen bestäubt.

\section{Bunte Beeren als Anpassung an Vogelausbreitung}

Die Früchte vieler Anthurien sind sehr attraktiv. Der Großteil der Arten wächst epiphytisch.

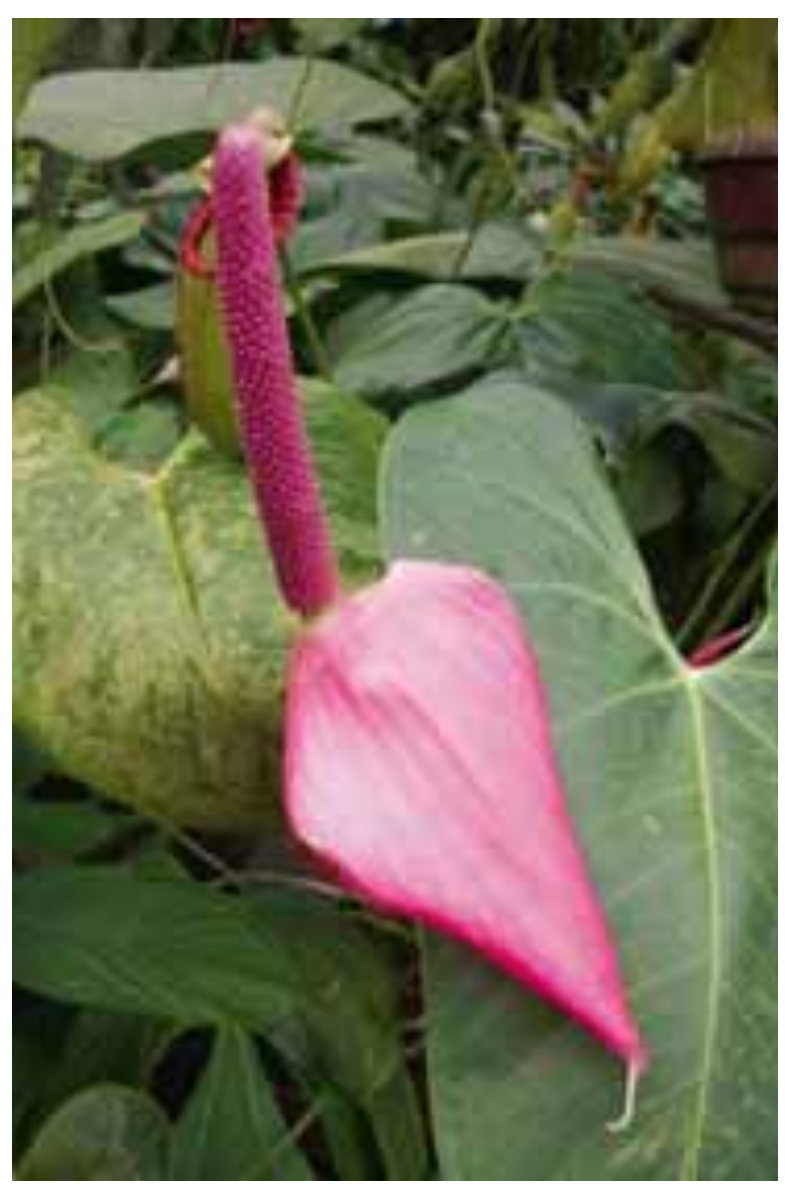

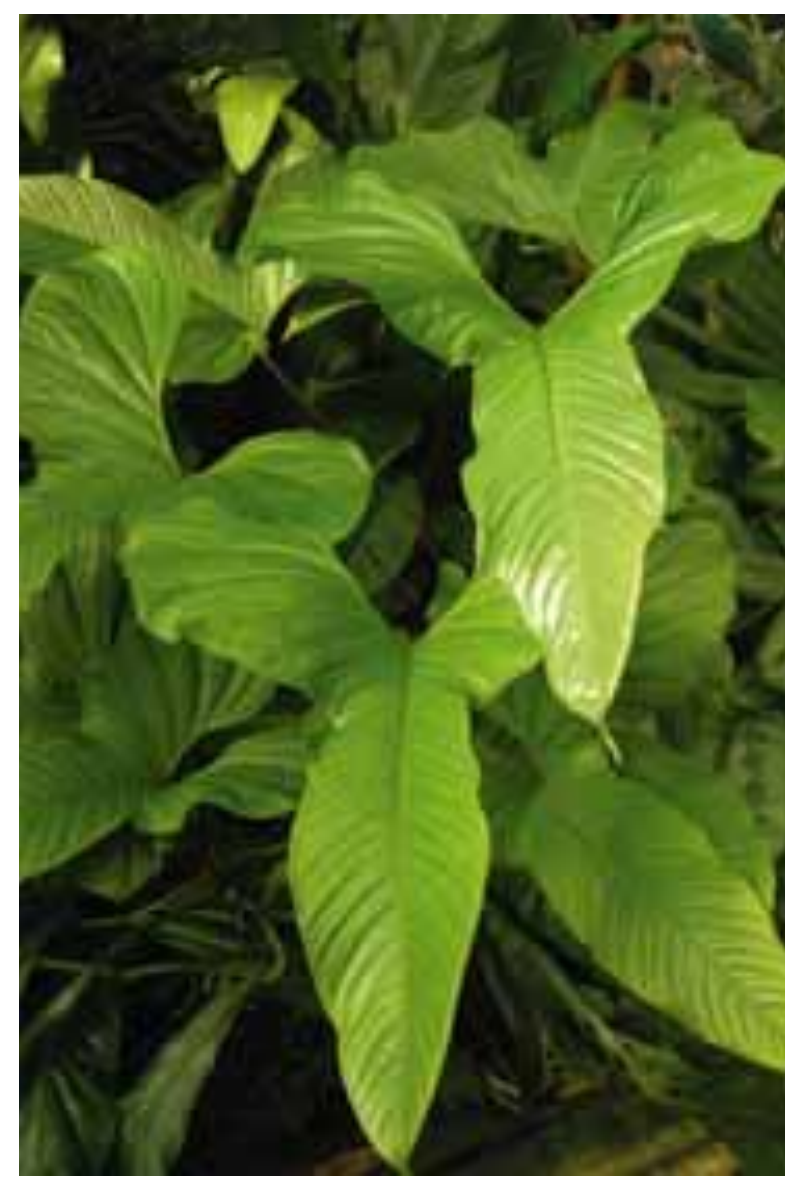

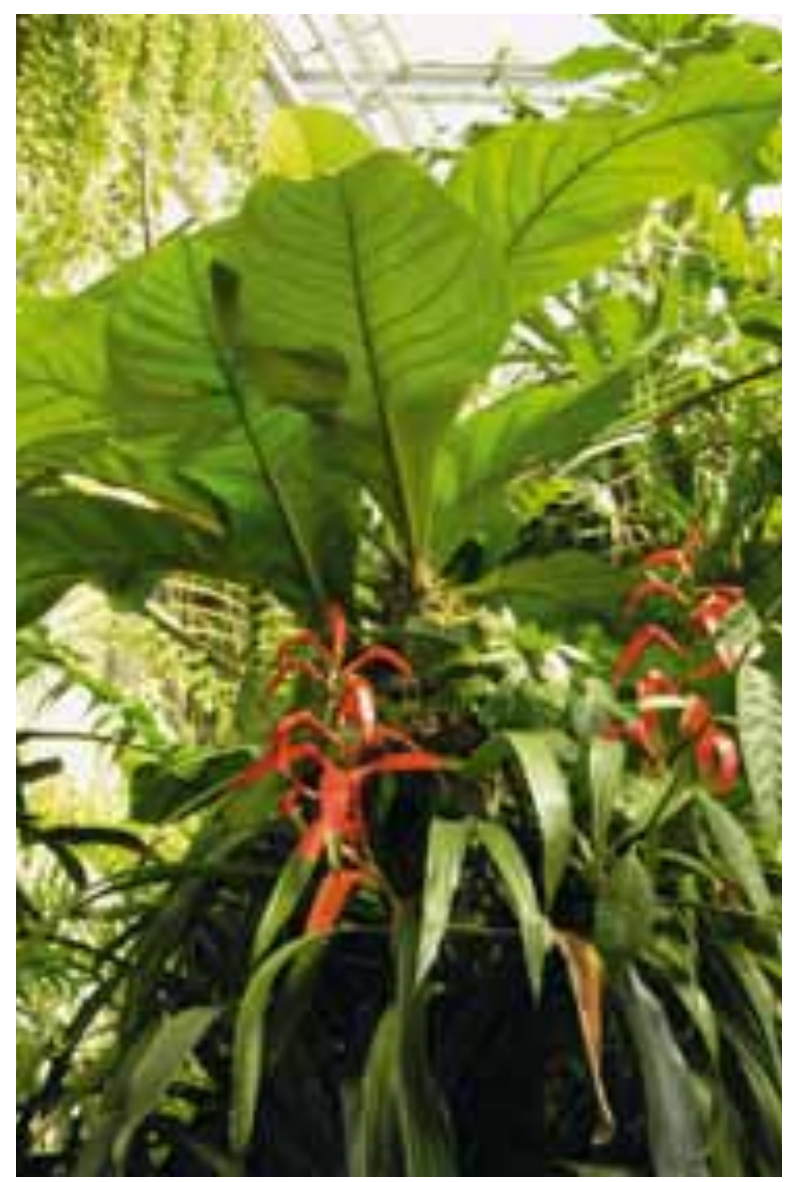




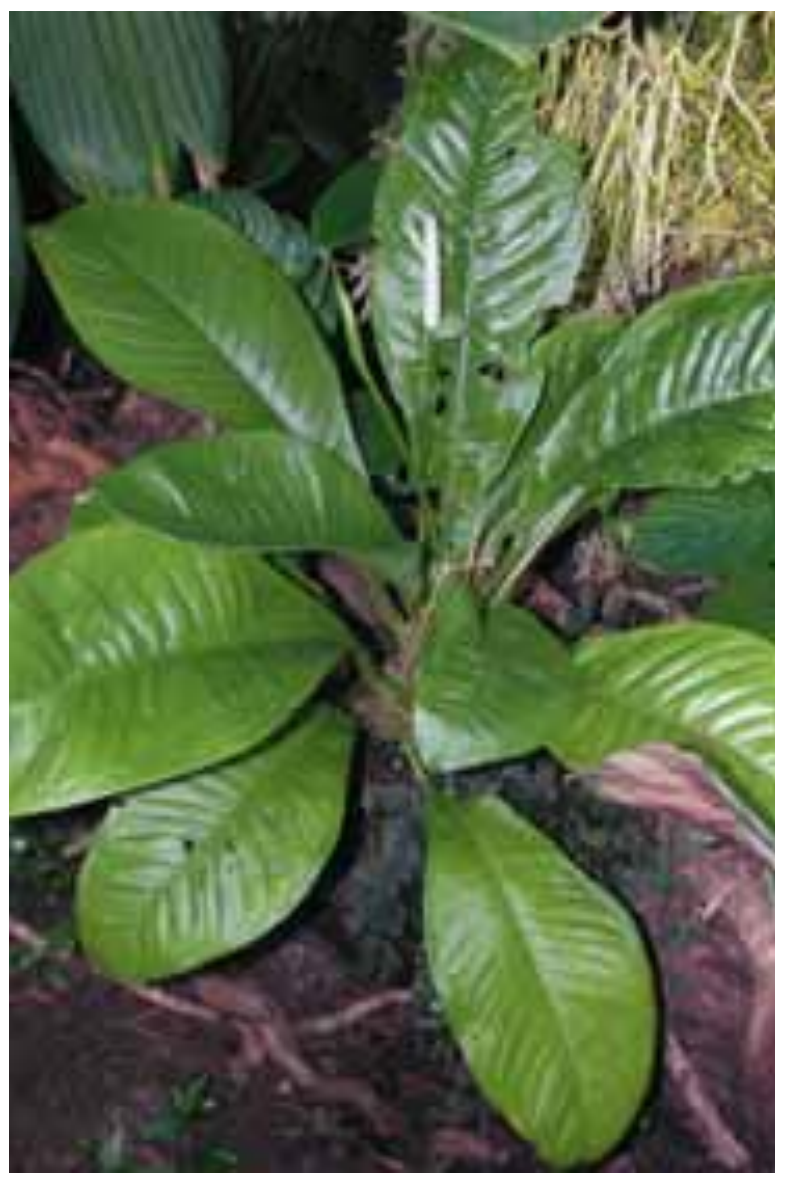

Darauf weisen schon die Wurzeln hin, die bei einigen Arten eine silbrig-weiße Hülle (Velamen) haben, ähnlich wie die Luftwurzeln der Orchideen. Aber auch die Früchte sind an die epiphytische Lebensweise angepasst und zeigen deutliche Anpassungen für Vogelausbreitung. Es sind Beeren von häufig leuchtender Farbe in Weiß, Gelb, Orange, Pink, Rot oder leuchtend Rosaviolett. Die Samen sind in weiches, schleimig-klebriges Fruchtfleisch eingebettet und erinnern so in der Konsistenz auffallend an die unserer Misteln. Vieles davon bleibt auch außen am Schnabel kleben und wird vom Vogel am nächsten Ast abgestreift.

Die Samen selber sind relativ groß und fleischig und keimen sofort. Ein Austrocknen vertragen sie nicht (daher ist es unsinnig, Anthurium-Saatgut z.B. in den Samenverzeichnissen botanischer Gärten anzubieten!). Oft keimen sie aber spontan aus den zerfallenden Früchten schon unter der eigenen Mutterpflanze. Relativ viele AnthuriumArten unserer Gewächshäuser sind selbstfertil, sodass die bunten Früchte ohne unser Zutun er-
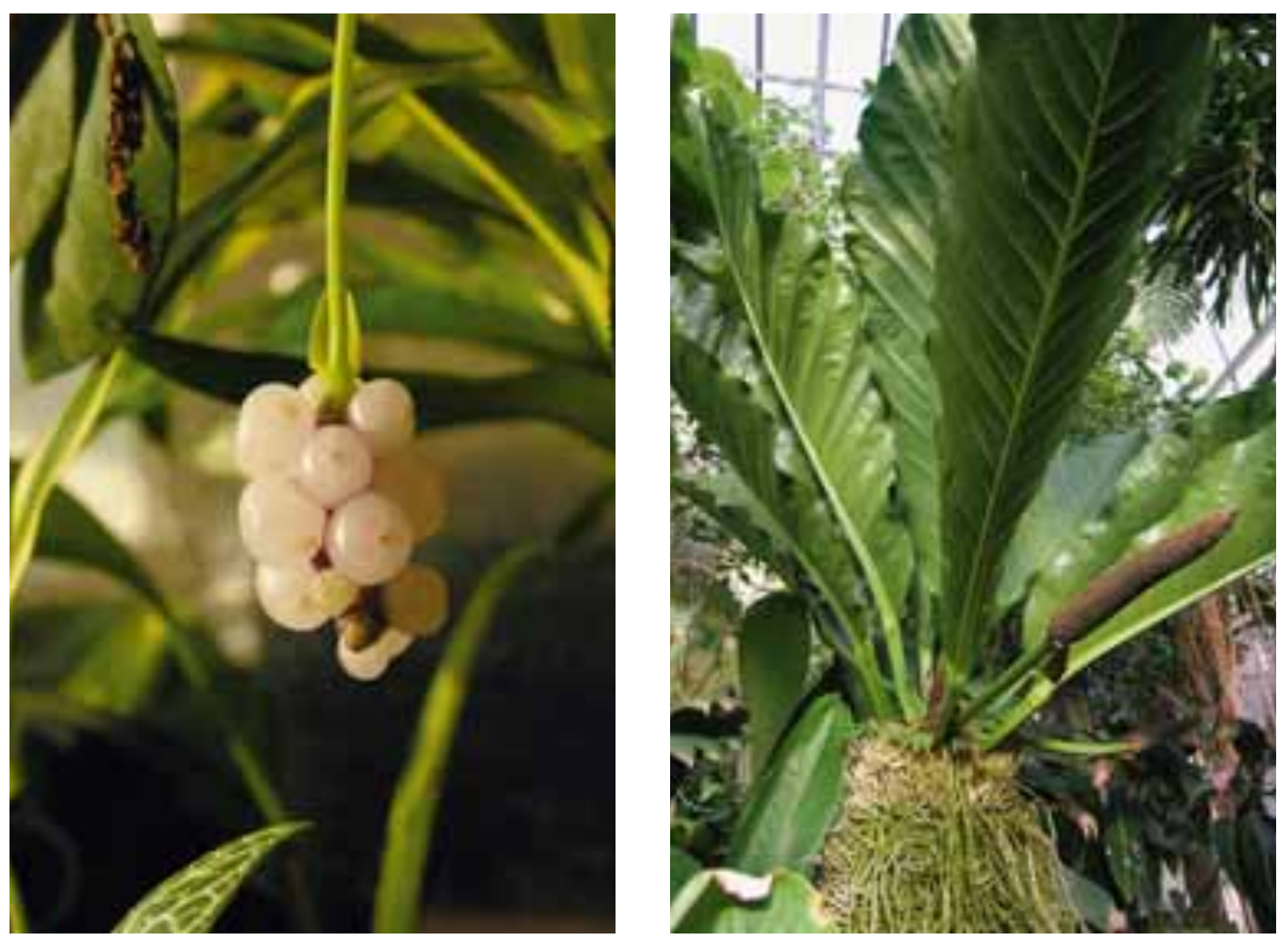
scheinen und sich die beschriebenen ausbreitungsökologischen Merkmale leicht studieren lassen.

\section{Anthurien-Vielfalt: Stehende und rankende, zierliche und gewaltige Formen}

Die größte Vielfalt der Anthurien zeigt sich jedoch in Wuchs und Blatt. Wenn auch viele Anthurien handliche, gedrungene Topfpflanzen darstellen, so gibt es doch Extreme: Einige Arten klettern mit meterlangen Trieben in die Baumkronen hinauf, so etwa Anthurium acrobates (Akrobat bedeutet aus dem Griechischen übersetzt Spitzenkletterer), $A$. sinuatum und $A$. digitatum. Solche Arten werden im Topf schnell zu mächtig und gedeihen am besten, wenn sie in den Schauhäusern ausgepflanzt werden. Demgegenüber stehen einige sehr kleinwüchsige epiphytische Arten wie $A$. gracile, A. scandens und $A$. trinerve. Die genannten Arten sind im gesamten tropischen Amerika weit verbreitet und gehören zur typischen Pflanzengesellschaft der Ameisengärten, z. B. zusammen mit Codonanthe crassifolia und bestimmten Aechmea-Arten. Alle drei Arten sind selbstfertil und fangen als Sämlinge schon „frühreif“ an, ihre unscheinbaren Blüten auszubilden, die aber von umso leuchtenderen Früchten gefolgt werden. Die drei Arten bilden üppige Luftwurzeln mit silberweißem Velamen aus. Bereits in 10er Ampeltöpfen sind diese Mini-Epiphyten ausgewachsen, blühen und fruchten. Es ist aber viel reizvoller, sie wie Orchideen ohne Substrat auf Brettchen oder Korkrinde zu kultivieren. Bei so magerer Kultur bleiben sie gedrungener, der Luftwurzelbesatz ist noch eindrucksvoller und sie blühen und fruchten ebenso gut.

Aber nicht alle Epiphyten sind klein und zierlich, auch nicht innerhalb der Gattung $A n$ thurium. Besonders beeindruckend sind die Anthurien der Untergattung Pachyneurium, bei denen die Blätter wie beim Nestfarn oder dem

Abb. 8 (Seite 16 oben): Anthurium oxycarpum.

Abb. 9 (Seite 16 unten links): Anthurium scandens var. pusillum, fruchtend.

Abb. 10 (Seite 16 unten rechts): Anthurium schechtendalii. Abb. 11 (oben): Anthurium schlechtendalii, Wurzeln.

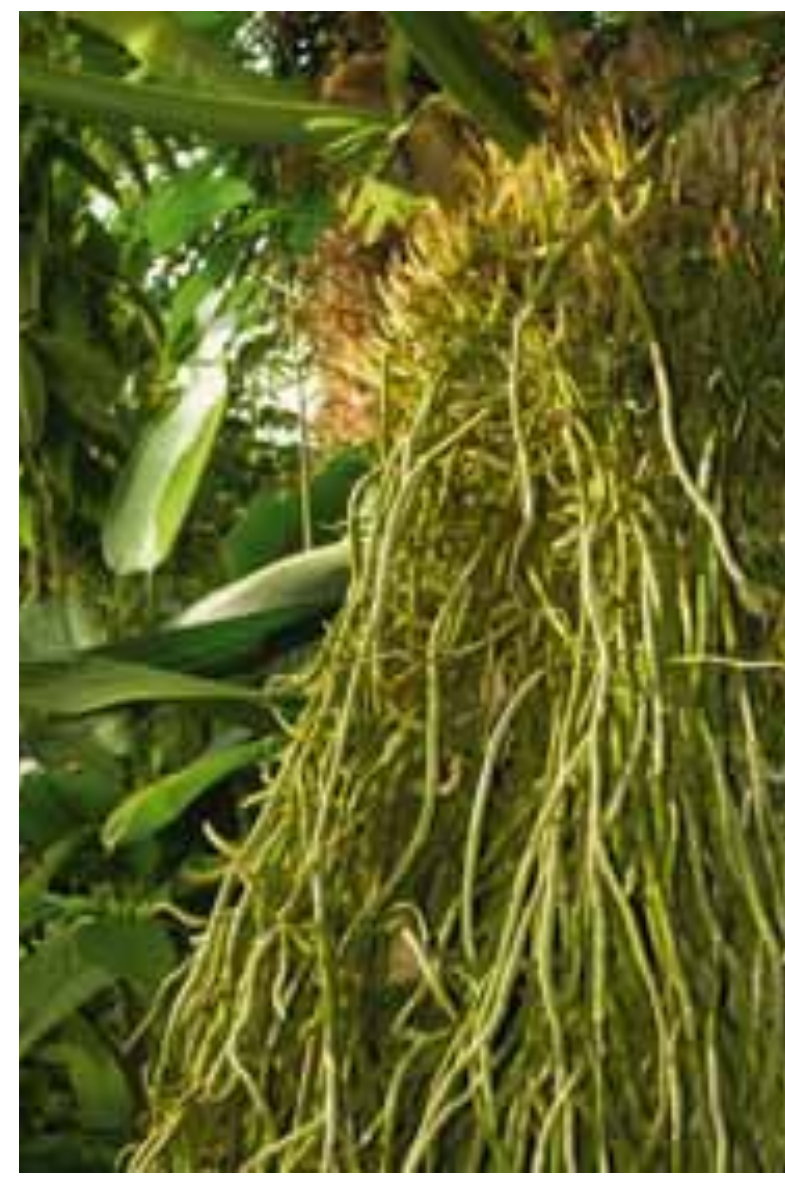

oben beschriebenen Philodendron campii einen regelmäßigen, humussammelnden Trichter formen, der oft von beachtlicher Größe ist. Das in Kultur am meisten verbreitete Anthurium dieses Typs ist $A$. hookeri, bei dem die Blatttrichter 1,5 m Durchmesser erreichen und mitsamt ihrem üppigen Luftwurzelwerk und ihrer $\mathrm{Hu}$ musfracht gut und gerne $20-30 \mathrm{~kg}$ schwer werden können. Noch viel gewaltiger, mit bis zu $3 \mathrm{~m}$ Spannweite, werden A. salviniae, A. harlingianum, A. loretense und $A$. schlechtendalii. Solche Arten werden übrigens unter dem Namen tabacón in Mittelamerika in ländlichen Gegenden als lebende „Öko-Tonnen“ genutzt. Man pflanzt sie in den Garten in einen alten Autoreifen und entsorgt alle organischen Abfälle in die Rosette (Uwe Scharf, pers. Mitteilung).

In unseren Gewächshäusern gehören solche gigantischen Anthurien zu den spektakulärsten Aronstabgewächsen, vor allem, wenn wir sie nicht in das Grundbeet pflanzen, sondern sie ihrem natürlichen Wuchs entsprechend aufgebockt als Epiphyten präsentieren. 

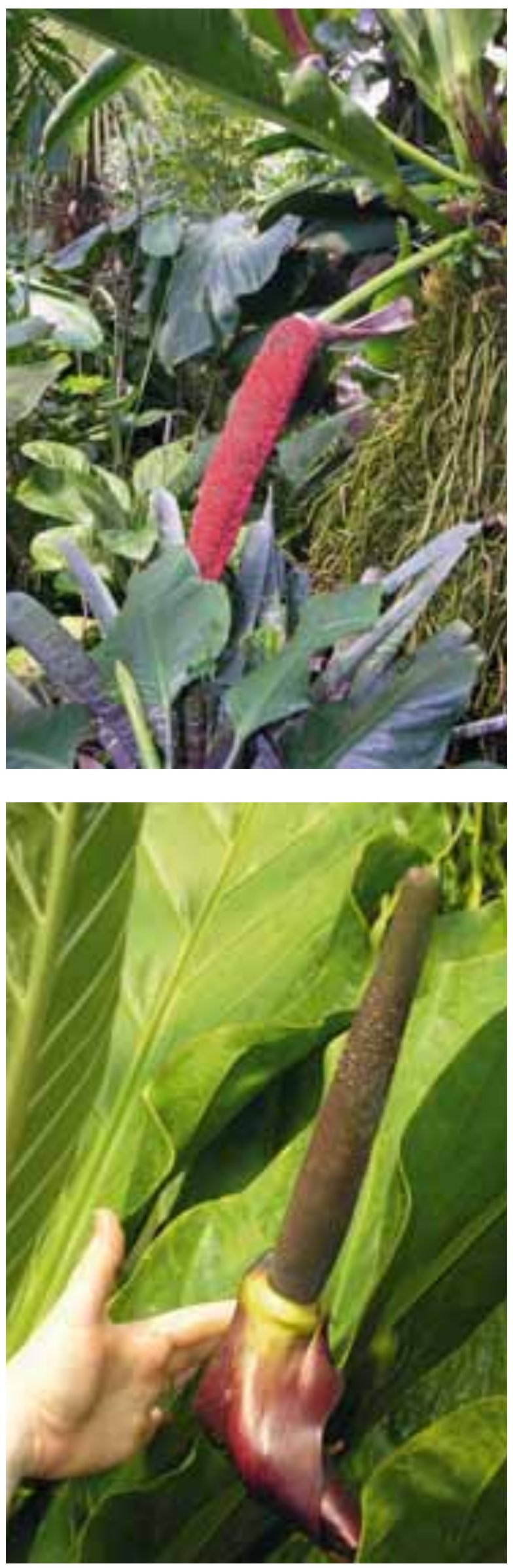

\section{Philodendren und Anthurien in öffentli- chen Schaugärten und in privater Kultur}

Der "Zimmerphilodendron" (Monstera deliciosa), ferner Philodendron bipinnatifidum, Ph. erubescens und $P h$. scandens gehören seit Jahrzehnten zum Standard-Grünpflanzensortiment. Sie halten relativ gut der trockenen Zimmerluft stand und, abgesehen von $P$. bipinnatifidum $\mathrm{mit}$ seinem dicken Stamm, lassen sich leicht, sogar im Wasserglas, durch Stecklinge vermehren. Philodendron scandens ist ein echter Winzling, der sich mit seinen hängenden Trieben gut als Ampelpflanze eignet. Philodendron erubescens, der mittlerweile auch in buntlaubigen Formen bzw. Hybriden im Handel erscheint, ist einer der wenigen „Philos", die im Zimmer sogar zur Blüte kommen.

Leider sind nicht alle für die Zimmerkultur geeignet. Viele sind Lianen, die die Baumkronen erklettern und für unsere Wohnverhältnisse schnell zu groß werden. Bei nicht wenigen misst die Blattspreite 50 bis $100 \mathrm{~cm}$ auf ebenso langem Stiel, sodass solche Arten am ehesten für tropische Schaugewächshäuser in Frage kommen, wo sie denselben Urwaldeindruck erzeugen wie auf den oben erwähnten romantischen Stahlstichen des 19. Jahrhunderts.

Unter den Arten mit schönen, fiederschnittigen und interessant geformten Blättern sind z. B. Philodendron bipennifolium, Ph. pedatum und $P h$. squamiferum zu nennen. Und außerdem gibt es auch Arten mit bunten Blättern: Bei Ph. mamei und Ph. ornatum (syn. Ph. sodiroi) ist das Blatt silbergrau gesprenkelt, bei $P h$. melanochrysum weist es auf dunkel-schwarzgrüner Oberfläche einen geheimnisvollen samtigen Schimmer auf, der noch durch eine golden glitzernde helle Blattnervatur unterbrochen wird. Ein ebensolches samtiges Funkeln hat die Blatt-

Abb. 12 (oben): Anthurium schlechtendalii, Fruchtstand.

Abb. 13 (unten): Anthurium schlechtendalii mit Größenvergleich.

Abb. 14 (Seite 19 oben): Anthurium veitchii.

Abb. 15 (Seite 19 unten links): Philodendron campii.

Abb. 16 (Seite 19 unten rechts): Philodendron mamei. 
oberfläche, auf smaragdgrünem Grund, bei Ph. verrucosum.

Diese schönen Philodendren sind von zahmerem Wuchs und hätten eigentlich „Fensterbankformat“. Es ist im Einzelnen auszuprobieren, ob sie der trockenen Zimmerluft standhalten.

Ganz ausgezeichnet eignen sich diese Philodendron-Schmuckstücke aber für schön bepflanzte Pflanzenvitrinen und „Regenwaldterrarien". Positiv ist hierbei, dass sie schon als ganz kleine Jungpflanzen die Schönheit ihrer Blätter zeigen und sich, sobald sie in der Vitrine zu groß werden, leicht durch Kopfstecklinge verjüngen lassen.

Auch die Gattung Anthurium, obwohl dreimal so artenreich wie Philodendron, hat für das Zimmer leider nur wenige Arten zu bieten. Unter den rot blühenden Flamingoblumen ist allein das mexikanische, hartblättrige $A$. scherzerianum zimmertauglich, während das an sich schönere $A$. andraeanum unter unseren Zimmerbedingungen oft trockene Blattränder und Schädlinge bekommt. Gelegentlich kommen auch Sämlinge

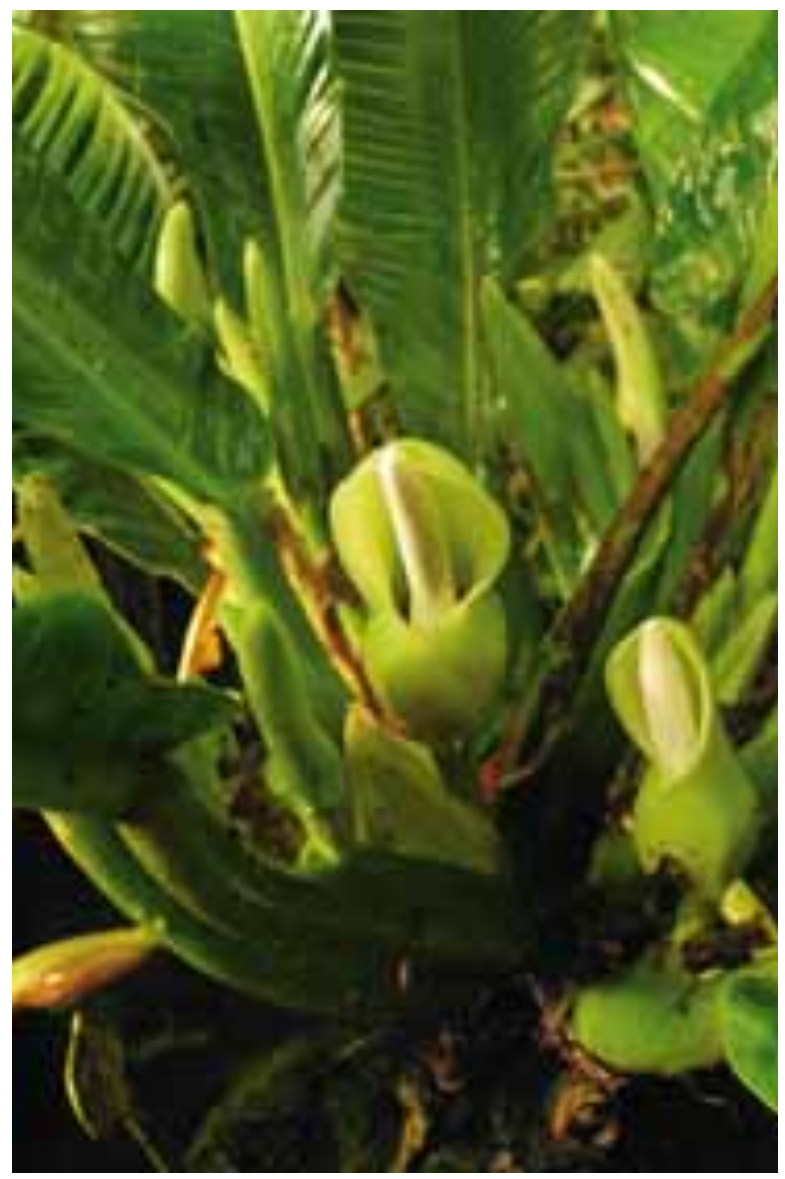

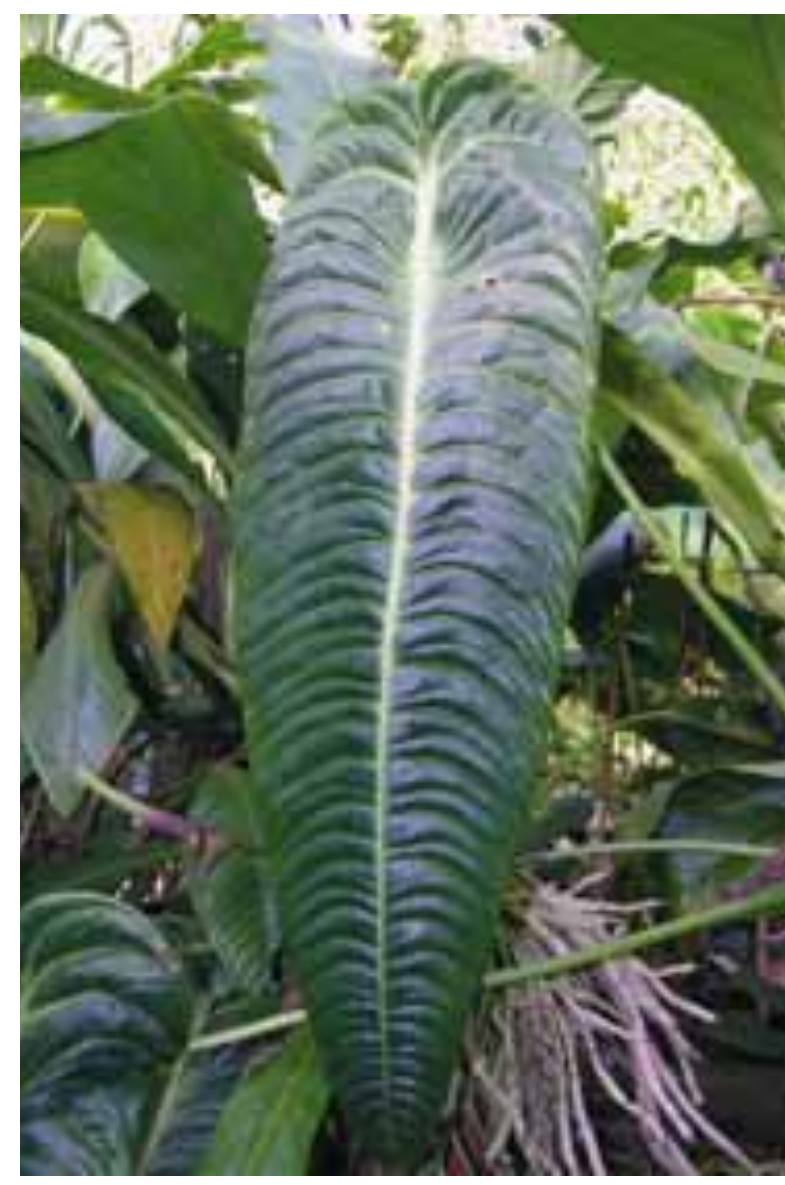

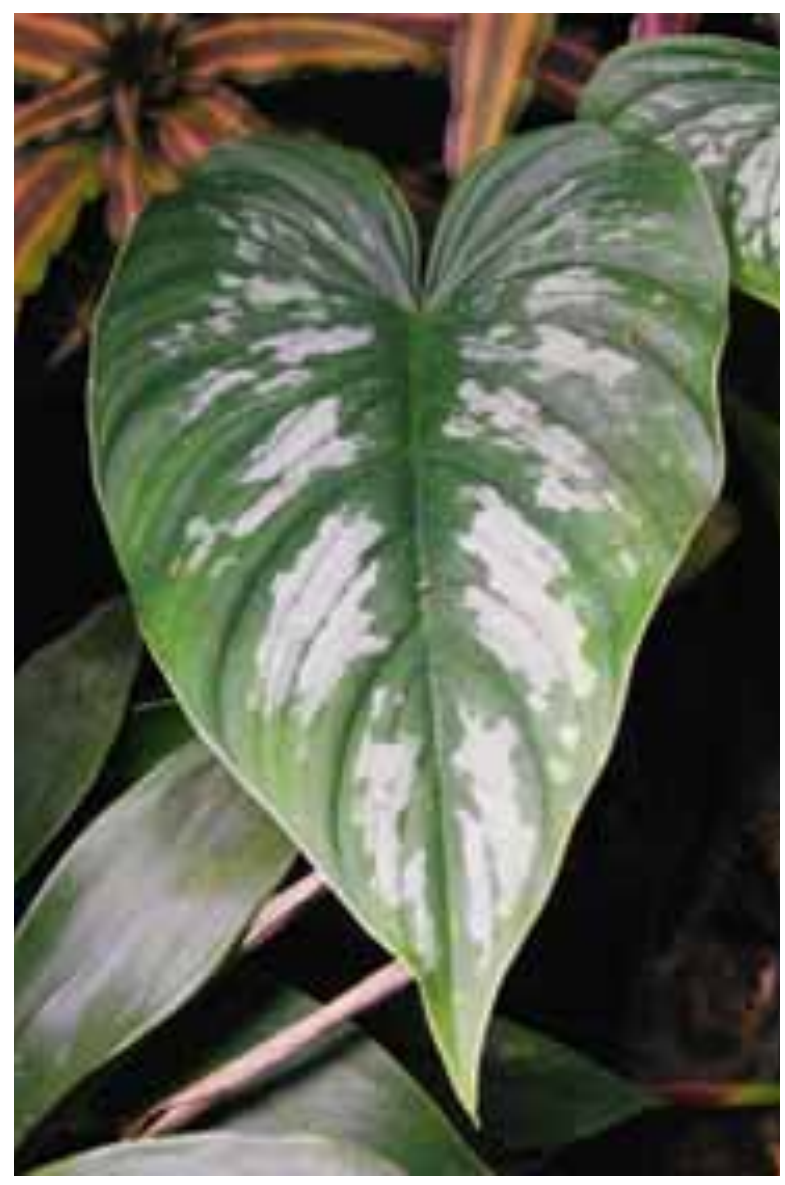



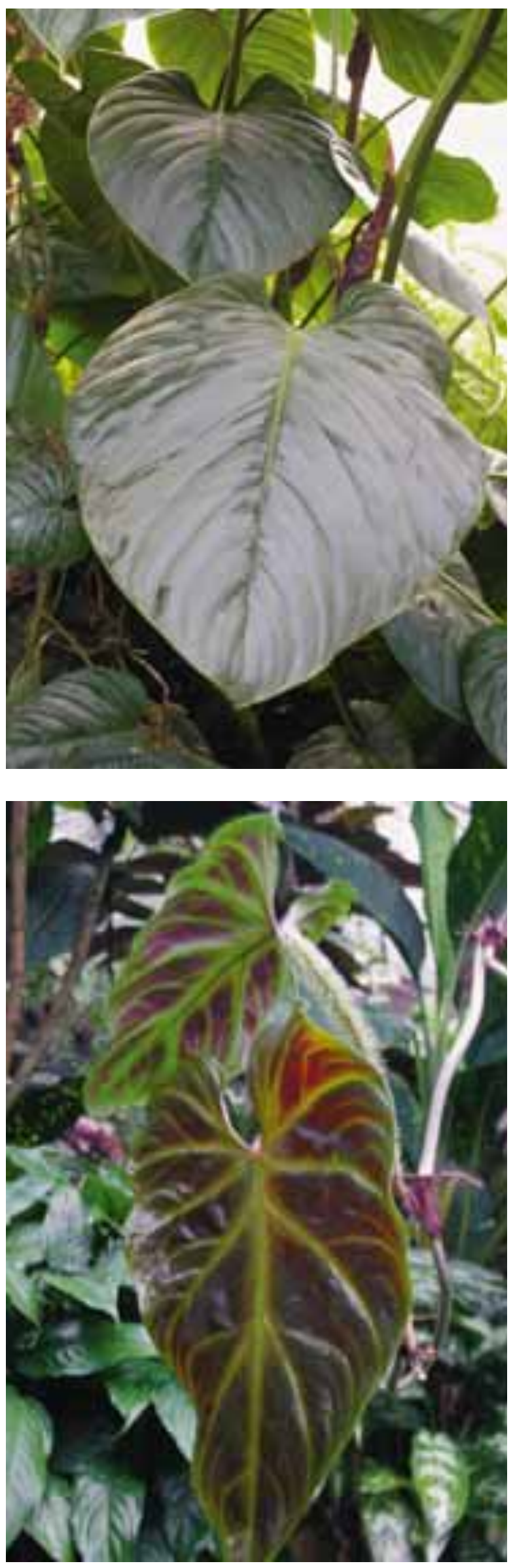

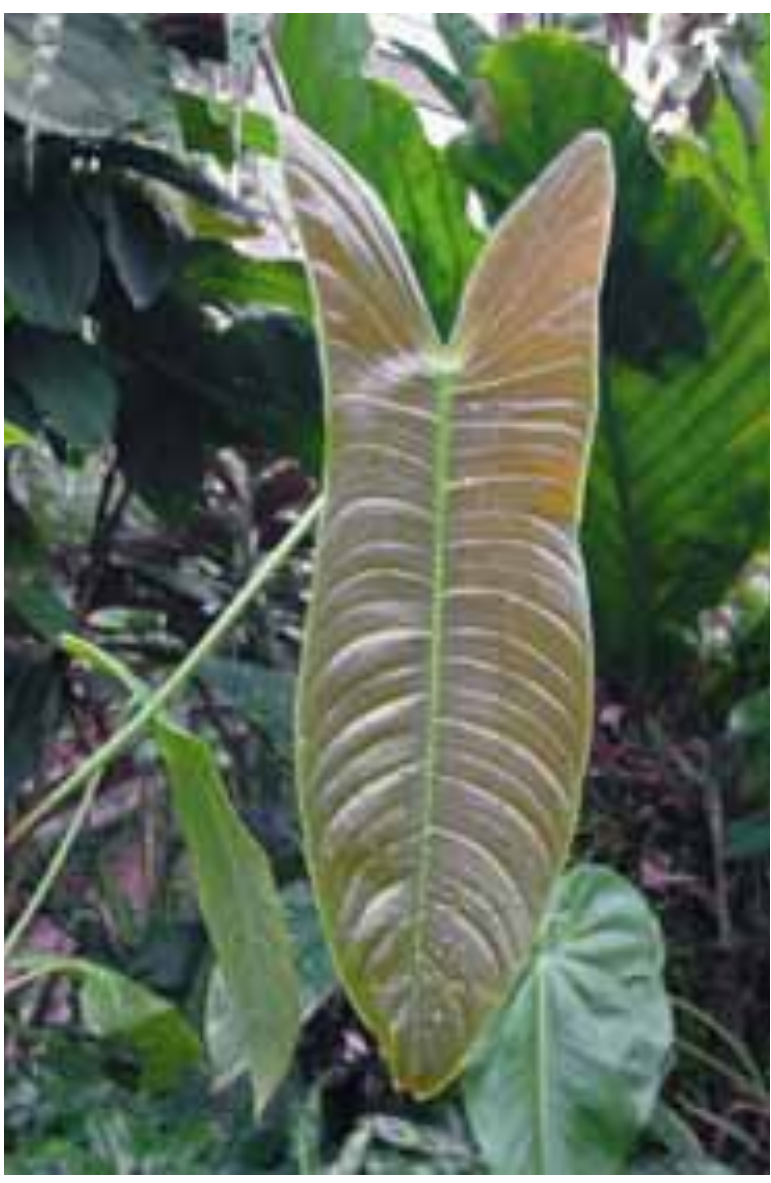

von $A$. crystallinum und $A$. clarinervium in den Handel, die auf ihrer breit-herzförmigen Spreite eine bezaubernde, silbrig glitzernde Nervatur zeigen, für deren Zimmereignung aber dasselbe gilt.

Dass die meterweit ausladenden und zentnerschweren Humusfänger der Untergattung Pachyneurium nicht für den Privathaushalt in Frage kommen, versteht sich von selbst. Es wurde allerdings oben schon erwähnt, dass die drei Liliput-Anthurien A. gracile, A. scandens und $A$. trinerve sich für Vitrinen und Terrarien eignen und schon als Mini-Sämlinge zu blühen und zu fruchten beginnen. Zusammen mit epiphytischen Bromelien, bodenbedeckenden Acanthaceen und Farnen lassen sich hier stimmungsvolle „Baumkronenwelten im Glase“ schaffen, in denen man z.B. tropische Frösche halten und zur Fortpflanzung bringen kann.

Abb. 17 (oben links): Philodendron ornatum.

Abb. 18 (oben rechts): Philodendron tenue.

Abb. 19 (unten): Philodendron verrucosum. 